\title{
Prática do autocuidado em prostitutas: aplicação do processo de enfermagem segundo a teoria de Orem
}

Recebido em: 20/12/2011

Aceito em: 15/02/2012
Isadora Marques Barbosa'

Cássia Fernandes Coelho' ${ }^{1}$

Priscila de Souza Aquino

Ana Karina Bezerra Pinheiro ${ }^{3}$

Desde tempos remotos, as prostitutas sofrem preconceitos. Esse fato pode acarretar dificuldades de atendimento às necessidades peculiares. Utilizar teoria de enfermagem no atendimento a prostitutas faz-se relevante por proporcionar maior comunicação terapêutica, adequando-se à problemática da paciente. Objetivou-se aplicar o processo de enfermagem utilizando a Teoria de Orem a prostitutas. Estudo descritivo, realizado por meio de três visitas domiciliárias a cinco prostitutas, utilizando um formulário estruturado. Os resultados mostraram os diagnósticos mais prevalentes: estilo de vida sedentário, 5 (26,3\%), manutenção ineficaz da saúde, 4 (21\%), e risco de solidão, 3 (15,7\%). Por meio da utilização da teoria, pôde-se investigar, detalhadamente, as alterações encontradas e traçar um plano de cuidados, bem como avaliar melhorias encontradas pós-intervenção. Concluiu-se que a associação de instrumentos da prática de enfermagem ao cuidado a essas mulheres é de grande valia para a promoção de sua saúde nas distintas interfaces.

Descritores: Autocuidado, Diagnóstico de Enfermagem, Prostituição.

\section{Self-Care practice in prostitutes: applying the nursing process according to the Orem's theory}

Since the inception of the profession, the prostitutes suffer prejudice. This may cause difficulties in meeting the unique needs. Using the theory of nursing care to prostitutes becomes relevant because it provides greater therapeutic communication, adapting it to the problem of the patient. The objective was to apply the nursing process using the Orem's theory to prostitutes. A descriptive study was carried out by means of three home visits to five prostitutes, using a structured form. The results showed the most prevalent diagnosis: Sedentary lifestyle 5 (26.3\%), Ineffective health maintenance 4 (21\%) and Risk for loneliness 3 (15.7\%). By using the theory, it was possible to investigate the changes, draw a plan of care and evaluate improvements after the intervention. It was concluded that the combination of instruments of nursing practice in caring for these women is of great value to the promotion of their health in different visions.

Descriptors: Self Care, Nursing Diagnosis, Prostitution.

\section{Practica del autocuidado en prostitutas: aplicacion del proceso de enfermería según la teoría de Orem}

Desde el inicio de la profesión, las prostitutas fueron víctimas de los prejuicios. Esto puede causar dificultades para atender sus necesidades. Usar la teoría de los cuidados de enfermería a las prostitutas se vuelve relevante porque proporciona mayor comunicación terapéutica y adaptación al problema del paciente. El objetivo fue aplicar el proceso de enfermería utilizando la teoría de Orem a prostitutas. Estudio descriptivo, hecho en el periodo de febrero a abril de 2009 , a través de tres visitas a domicilio a cinco prostitutas, utilizando un formulario estructurado. Los resultados mostraron los diagnósticos más frecuentes: Estilo de vida sedentario 5 (26,3\%), Mantenimiento de la salud ineficaces 4 (21\%) y Riesgo de soledad 3 (15,7\%). Mediante el uso de la teoría puede ser investigado en detalle los cambios observados, elaborar un plan de atención, y evaluar mejoras después de la intervención. Se concluyó que la combinación de instrumentos de la práctica de enfermería en el cuidado de estas mujeres es de gran valor para la promoción de su salud en diferentes interfaces.

Descriptores: Autocuidado, Diagnóstico de Enfermería, Prostitución.

\section{INTRODUÇÃO}

F alar sobre sexualidade ainda constitui um tabu social, mesmo com os avanços na discussão dessa temática e as mudanças na educação familiar. Além disso, a expressão da sexualidade ainda perpassa por valores e significados transmitidos a cada geração, o que pode ocasionar preconceitos e estigmas a pessoas que fogem às regras.

Vários autores tentam atribuir um significado à prostituição. $\mathrm{O}$ termo prostituta pode ser definido como "mulher que pratica o ato sexual por dinheiro"(1). Já ao termo prostituição, tem-se: "troca consciente de favores sexuais por interesses não sentimentais ou afetivos" ${ }^{\prime \prime 2}$. Outros autores acrescentam que a prostituição pode ser definida como o conjunto de pessoas ou instituições que promovem ou realizam relações sexuais com o objetivo de satisfação fisiológica, psíquica ou mesmo econômica, na qual estão excluídos sentimentos como o amor ${ }^{(3)}$.

Diversos são os motivos que podem levar uma mulher

1 Enfermeira formada pela Universidade Federal do Ceará. E-mail: isadoramarx@gmail.com.

2 Enfermeira, doutora em enfermagem, professora-assistente da Universidade Federal do Piauí, Picos.

3 Enfermeira, doutora em enfermagem, professora adjunta IV do departamento de enfermagem da Universidade Federal do Ceará. 
à prática da prostituição, entre eles: residência em regiões caracterizadas por problemas sociais, violência estrutural, privação econômica ou responsabilidade familiar, baixa escolaridade, abandono ou negligências familiares, violência sexual, psicológica ou doméstica ${ }^{(4,5)}$.

Desde o surgimento da profissão, as prostitutas sofrem preconceitos e são acusadas de disseminar doenças, além de ferir as regras dos costumes sociais. Estigmas são impostos de maneira brutal sobre essas mulheres, que utilizam o sexo com finalidade lucrativa. Devido a isso, as prostitutas podem sofrer discriminação pela população ou até mesmo por alguns profissionais de saúde, que focam o atendimento a essas mulheres mais nos fatores de risco, esquecendo-se de fornecer uma atenção integral(6). Deve-se repensar a qualidade da assistência a essas mulheres ${ }^{(7)}$.

Dessa forma, o fato de essas mulheres terem de cuidar de sua saúde sem, muitas vezes, instruções apropriadas pode dificultar a prática do autocuidado. Tendo em vista a importância dessa atividade para a saúde dos indivíduos, faz-se necessário o uso de um arcabouço teórico para a investigação e a aplicação do cuidado. A Teoria do Autocuidado de Orem discorre sobre uma série de requisitos importantes para essa prática, além de orientar o profissional de enfermagem a promover um maior nível de autocuidado para o paciente.

Partindo do exposto, decidiu-se realizar o estudo com o objetivo de aplicar o processo de enfermagem, baseado na Teoria do Autocuidado de Orem, em prostitutas.

\section{REFERENCIALTEÓRICO}

A Teoria de Orem proporciona ao indivíduo o autocuidar,

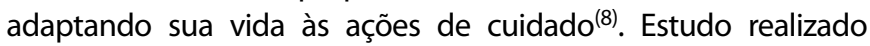
utilizando a teoria do autocuidado mostrou que seu uso propiciou uma comunicação terapêutica entre a enfermeira e a paciente, adequando-se à problemática da paciente $\mathrm{e}^{(9)}$.

Segundo a Teoria de Orem, autocuidado constitui atividades em que o indivíduo tem plena sabedoria para desenvolvê-las e executá-las em benefício próprio, para que tenha uma manutenção do bem-estar, da saúde e da vida ${ }^{(10)}$.

$\mathrm{Na}$ prática do autocuidado, tem-se uma interação do profissional de saúde com o paciente para a detecção de problemas e possíveis intervenções. É fundamental a participação do paciente na formulação do plano de intervenções, pois o profissional de saúde irá guiá-lo na prática dos cuidados, fazendo com que ele tenha cada vez mais independência ${ }^{(9,11)}$.

Os requisitos do autocuidado apresentados por Orem são universais, de desenvolvimento e referentes ao desvio de saúde. Os requisitos referidos são ações voltadas para prover autocuidado. Os universais, como evidenciado na literatura, são associados a processos de vida, influenciando diretamente a estrutura do ser humano e suas funções, como suprimento adequado de água e de alimentos. Os de desenvolvimento representam os estágios do ciclo vital, incluindo algumas condições ou eventos associados por exemplo, a adaptação a um novo trabalho. Os de desvio de saúde são denotados em condições de doenças que podem vir a gerar dificuldades na prática do autocuidado ${ }^{(10)}$.
Para aprimorar o cuidado do paciente, Orem propõe três momentos ${ }^{(10)}$ :

No primeiro momento, o profissional de enfermagem deve interagir com o paciente e, a partir de então, coletar os dados que precisa para fazer os diagnósticos adequados e as intervenções precisas.

No segundo momento, o profissional de enfermagem vai aprimorar o plano de cuidado e passar a colocá-lo em prática, com a ajuda dos membros da família ou responsáveis pelo cuidado. Avalia-se nessa hora o potencial do paciente para a prática do autocuidado.

No terceiro momento, têm-se a preparação do paciente, da família ou do responsável para a prática do autocuidado sem a intervenção do profissional de enfermagem, tornando-os independentes. Aqui, são documentadas as ações estabelecidas de acordo com a demanda de autocuidado.

A Teoria de Orem torna-se mais aplicável quando embasada pelo processo de enfermagem, que poderá sistematizar as ações a serem realizadas. Além disso, o processo permite a detecção de problemas reais ou potenciais de saúde e intervenções direcionadas às necessidades observadas, o que poderá ser mais eficaz para a melhora do nível de saúde do paciente ${ }^{(12)}$.

O processo de enfermagem é iniciado com uma investigação que consta da coleta de dados, seguido pelo diagnóstico de enfermagem. Depois, é feito um planejamento constando de intervenções que serão implementadas. É um processo que prioriza o cuidado humanizado, respeitando as individualidades e os valores de cada pessoa. O profissional de enfermagem deve fazer uso desse processo, pois só assim conseguirá realizar um cuidado holístico, respeitando e valorizando não apenas o biológico $(8,12)$.

Se associarmos o processo de enfermagem com a Teoria do Autocuidado de Orem, teremos a determinação das deficiências na prática do autocuidado e, assim, o profissional de enfermagem poderá intervir apropriadamente ${ }^{(10)}$.

\section{MATERIAL E MÉTODO}

Trata-se de um estudo descritivo com abordagem qualitativa. A abordagem qualitativa permite enfocar as diferentes experiências vividas, resultado das relações e dos significados do contexto dos diversos cenários naturais ${ }^{(13)}$. Já as pesquisas descritivas pretendem informar as características dos fenômenos ou das relações entre as variáveis ${ }^{(14)}$.

Decidiu-se realizar o estudo com prostitutas atuantes em ambientes fechados de Fortaleza. O local selecionado foi o centro da cidade e um bairro da periferia. Entrevistaram-se cinco prostitutas por conveniência, com utilização de um formulário de entrevista baseado na Teoria do Autocuidado de Orem, focando os requisitos universais, os de desenvolvimento (relacionados à adaptação dessas mulheres a um novo estilo de vida) e os de desvio de saúde (focando as condições de saúde que possam influenciar a prática do autocuidado).

Os dados foram coletados no período de março a abril de 2009. No primeiro momento, foram detectadas as alterações no autocuidado por meio da aplicação do referido instrumento. Posteriormente, identificaram-se os diagnósticos de enfermagem 
segundo a Taxonomia II da North American Nursing Diagnosis Association (Nanda) ${ }^{(15)}$. Para cada diagnóstico selecionado, fez-se uso das intervenções contidas na Classificação das Intervenções de Enfermagem (NIC) ${ }^{(16)}$ para nortear o plano de cuidados.

No segundo momento, o plano de cuidados construído foi apresentado e reformulado junto com as prostitutas, para abordar de forma mais direcionada as necessidades das pacientes.

O terceiro momento foi utilizado para avaliar a eficácia das intervenções, bem como se essas mulheres estavam aptas a realizar as atividades de autocuidado que poderiam influenciar sua saúde. Esse último encontro objetivou avaliar o grau de independência para a realização do autocuidado.

Os dados oriundos das visitas foram discutidos de acordo com a literatura pertinente e agrupados segundo as categorias de autocuidado que surgiram. A pesquisa atendeu à resolução $n^{\circ}$ 196/1996, que trata das pesquisas envolvendo seres humanos, e foi aprovada pelo Comitê de Ética em Pesquisa da Universidade Federal do Ceará, sob o protocolo n 261/2008.

\section{RESULTADOS}

A partir dos achados, foi possível retratar os principais diagnósticos encontrados, com seus respectivos planos e intervenções. Os diagnósticos foram ainda classificados de acordo com a Nanda.
Segundo a associação, os diagnósticos reais descrevem respostas humanas a condições atuais de saúde/processo de vida que existem em um indivíduo. Já os diagnósticos de risco caracterizam uma resposta humana a condições de saúde/processos de vida que podem se desenvolver. Os diagnósticos de promoção da saúde são definidos pela motivação e desejo de uma pessoa de aumentar o bem-estar e concretizar o potencial de saúde humana, melhorando com isso sua qualidade de vida ${ }^{(15,17)}$.

Após o primeiro momento junto às prostitutas, identificaramse os possíveis diagnósticos e intervenções de enfermagem que norteariam a elaboração do plano de cuidados. Verificou-se a prevalência dos diagnósticos reais, o que já era esperado por se tratar de um grupo bastante vulnerável a problemas. As alterações relacionadas aos requisitos de desenvolvimento estiveram presentes nos achados, principalmente por se tratar de pessoas em uma situação de vida peculiar, na qual há necessidades distintas, comuns à fase de vida em que se encontram. O sistema apoio-educação foi o mais utilizado, uma vez que as pacientes apresentavam independência em seu autocuidado, necessitando apenas de orientação e apoio no desenvolvimento dessa prática. Esses dados foram dispostos na tabela 1.

Estiveram ainda presentes outros diagnósticos de enfermagem, porém detectados com menor frequência. Entre

TABELA 1 - Distribuição dos principais diagnósticos de enfermagem e plano de cuidados encontrados nas visitas domiciliárias a prostitutas. Fortaleza-CE, abr/mai, 2009

\begin{tabular}{|c|c|c|c|}
\hline $\begin{array}{l}\text { REQUISITOS DE } \\
\text { AUTOCUIDADO } \\
\text { ALTERADOS }\end{array}$ & $\begin{array}{l}\text { DIAGNÓSTICO } \\
\text { DE ENFERMAGEM }\end{array}$ & PLANO & INTERVENÇÕES \\
\hline Universais & $\begin{array}{l}\text { Estilo de vida sedentário } \\
\text { relacionado a falta de } \\
\text { motivação evidenciada por } \\
\text { escolha de rotina diária sem } \\
\text { exercícios físicos }\end{array}$ & $\begin{array}{l}\text { Meta: manter o paciente informado } \\
\text { sobre os beneficios do exercício físico } \\
\text { Objetivo: promover orientações que } \\
\text { facilitem a adesão do paciente à rotina } \\
\text { de exercícios } \\
\text { Sistema de enfermagem: } \\
\text { apoio-educação }\end{array}$ & $\begin{array}{l}\text { - Orientar a paciente quanto aos } \\
\text { benefícios do exercício físico } \\
\text { - Informá-la acerca do tempo mínimo } \\
\text { de } 20 \text { minutos, três vezes por semana, } \\
\text { para benefícios sistêmicos }\end{array}$ \\
\hline Universais & $\begin{array}{l}\text { Nutrição desequilibrada: } \\
\text { menos do que as } \\
\text { necessidades corporais } \\
\text { relacionadas a fatores } \\
\text { psicológicos evidenciados } \\
\text { por conceitos errados }\end{array}$ & $\begin{array}{l}\text { Meta: promover o restabelecimento } \\
\text { de uma alimentação condizente com } \\
\text { as necesidades da paciente } \\
\text { Objetivo: promover orientações } \\
\text { acerca de tipo e frequência da } \\
\text { alimentação mais indicada } \\
\text { Sistema de enfermagem: } \\
\text { apoio-educação }\end{array}$ & $\begin{array}{l}\text { - Incentivar a ingestão de } \\
\text { alimentos leves entre as refeições } \\
\text { principais } \\
\text { - Orientar a ingestão de uma } \\
\text { dieta balanceada, rica em frutas } \\
\text { e verduras, livre de alimentos } \\
\text { gordurosos }\end{array}$ \\
\hline Universais & $\begin{array}{l}\text { Nutrição desequilibrada: } \\
\text { mais do que as } \\
\text { necessidades corporais } \\
\text { relacionadas à ingestão } \\
\text { excessiva em relação às } \\
\text { necessidades metabólicas, } \\
\text { evidenciada por nível de } \\
\text { atividade sedentário }\end{array}$ & $\begin{array}{l}\text { Meta: promover a adequação da } \\
\text { paciente a uma alimentação saudável } \\
\text { Objetivo: orientar a adoção de uma } \\
\text { dieta saudável } \\
\text { Sistema de enfermagem: } \\
\text { Apoio-educação }\end{array}$ & $\begin{array}{l}\text { - Incentivar a ingestão de alimentos } \\
\text { com menor teor de carboidrato } \\
\text { - Orientar a ingestão de frutas entre } \\
\text { as refeições } \\
\text { - Reforçar a prática de atividade } \\
\text { física }\end{array}$ \\
\hline
\end{tabular}




\begin{tabular}{|c|c|c|c|}
\hline $\begin{array}{l}\text { REQUISITOS DE } \\
\text { AUTOCUIDADO } \\
\text { ALTERADOS }\end{array}$ & $\begin{array}{l}\text { DIAGNÓSTICO } \\
\text { DE ENFERMAGEM }\end{array}$ & PLANO & INTERVENÇŌES \\
\hline Desenvolvimento & $\begin{array}{l}\text { Desobediência relacionada } \\
\text { a forças motivacionais } \\
\text { evidenciada por } \\
\text { comportamento indicativo } \\
\text { de falta de adesão }\end{array}$ & $\begin{array}{l}\text { Meta: aumentar a adesão da } \\
\text { paciente aos programas de saúde } \\
\text { Objetivo: incentivar a adesão a } \\
\text { medidas de promoção da saúde } \\
\text { Sistema de enfermagem: } \\
\text { apoio-educação }\end{array}$ & $\begin{array}{l}\text { - Realizar atividades de educação } \\
\text { para saúde relacionada à promoção } \\
\text { da adesão } \\
\text { - Apoiar a tomada de decisões da } \\
\text { paciente enfatizando os riscos e } \\
\text { benefícios }\end{array}$ \\
\hline Desenvolvimento & $\begin{array}{l}\text { Manutenção ineficaz da } \\
\text { saúde relacionada com o } \\
\text { enfrentamento individual } \\
\text { ineficaz evidenciado por } \\
\text { incapacidade de assumir } \\
\text { a responsabilidade de } \\
\text { atender a práticas básicas } \\
\text { de saúde }\end{array}$ & $\begin{array}{l}\text { Meta: restabelecer a manutenção } \\
\text { da saúde da paciente } \\
\text { Objetivo: promover orientações de } \\
\text { saúde que facilitem a mudança de } \\
\text { comportamento } \\
\text { Sistema de enfermagem: } \\
\text { apoio-educação }\end{array}$ & $\begin{array}{l}\text { - Apoiar a tomada de decisões } \\
\text { - Incentivar a redução e posterior } \\
\text { abstenção do fumo } \\
\text { - Incentivar a participação em } \\
\text { grupos de apoio } \\
\text { - Orientar a execução de atividades } \\
\text { que ajudem a minimizar a } \\
\text { ansiedade }\end{array}$ \\
\hline Desenvolvimento & $\begin{array}{l}\text { Risco de infecção } \\
\text { relacionado ao não-uso } \\
\text { de preservativo com o } \\
\text { parceiro fixo }\end{array}$ & $\begin{array}{l}\text { Meta: diminuir o risco de infecção } \\
\text { Objetivo: aumentar a proteção } \\
\text { contra infecções } \\
\text { Sistema de enfermagem: } \\
\text { apoio-educação }\end{array}$ & $\begin{array}{l}\text { - Orientar a utilização do } \\
\text { preservativo masculino em todas } \\
\text { as relações } \\
\text { - Esclarecer os riscos de transmissão } \\
\text { de DST/HIV em cada prática sexual } \\
\text { - Incentivar a prática do banho após } \\
\text { as relações sexuais }\end{array}$ \\
\hline Desenvolvimento & $\begin{array}{l}\text { Risco de baixa autoestima } \\
\text { situacional relacionado a } \\
\text { fracassos, mudanças de } \\
\text { papel social, rejeições e } \\
\text { falta de reconhecimento }\end{array}$ & $\begin{array}{l}\text { Meta: promover a elevação da } \\
\text { autoestima } \\
\text { Objetivo: incentivar a adoção } \\
\text { de atitudes que propiciem } \\
\text { melhora na autoestima } \\
\text { Sistema de enfermagem: } \\
\text { apoio-educação }\end{array}$ & $\begin{array}{l}\text { - Apoiar a procura por redes de } \\
\text { apoio, como grupos e associações } \\
\text { - Identificar pessoas significativas } \\
\text { que possam contribuir para a } \\
\text { melhoria da autoestima } \\
\text { - Orientar a realização de outras } \\
\text { atividades nos períodos livres }\end{array}$ \\
\hline Desenvolvimento & $\begin{array}{l}\text { Disposição para aumento } \\
\text { do autocuidado } \\
\text { evidenciado por expressar } \\
\text { desejo de aumentar } \\
\text { a independência na } \\
\text { manutenção da saúde }\end{array}$ & $\begin{array}{l}\text { Meta: potencializar o desejo } \\
\text { pelo autocuidado } \\
\text { Objetivo: orientar medidas de } \\
\text { promoção do autocuidado } \\
\text { Sistema de enfermagem: } \\
\text { apoio-educação }\end{array}$ & $\begin{array}{l}\text { - Incentivar a adoção de } \\
\text { práticas de autocuidado } \\
\text { junto à paciente } \\
\text { - Orientar acerca dos } \\
\text { benefícios das práticas de } \\
\text { promoção da saúde }\end{array}$ \\
\hline
\end{tabular}

eles, destacam-se: controle ineficaz do regime terapêutico relacionado com exigências excessivas evidenciado por escolhas da vida diária ineficazes para atingir os objetivos de saúde; comportamento de saúde propenso a risco relacionado à atitude negativa em relação aos cuidados de saúde evidenciado por minimizar a mudança no estado de saúde e por não conseguir agir de forma a prevenir problemas de saúde; manutenção ineficaz da saúde relacionada com enfrentamento individual ineficaz e evidenciada por história de ausência de comportamento de busca da saúde; nutrição desequilibrada: menos do que as necessidades corporais relacionada a fatores psicológicos e evidenciada por perda de peso com ingestão adequada de comida e por peso corporal abaixo do ideal; e risco de solidão relacionado a privação afetiva.

Quanto aos fatores relacionados mais prevalentes nos diagnósticos, percebeu-se a presença de fatores psicológicos, como: enfrentamento individual, privação afetiva, atitude negativa em relação aos cuidados de saúde, entre outros. Com relação às características definidoras, as mesmas incluíram: conceitos errados, escolha de uma rotina diária sem exercícios 
físicos, história de ausência de comportamento de busca de saúde, desejo de aumentar o conhecimento de estratégias de autocuidado e expressão de desejo de aumentar o autocuidado. Ou seja, para uma adequada intervenção de saúde, seria necessária a abordagem educativa, com a participação efetiva dos sujeitos, propiciando conhecimento, empoderamento e mudança de comportamento.

Durante a primeira interação, as mulheres demonstraram bastante receio e medo por participar deste trabalho. No entanto, no decorrer da entrevista, foram se sentindo mais à vontade para responder às indagações. Ao fim do encontro, percebeu-se a satisfação das entrevistadas por meio de relatos.

$\mathrm{Na}$ segunda visita, também realizada no local de trabalho, discutiu-se o plano de intervenção elaborado pelas pesquisadoras e adaptado de acordo com as reais necessidades de cada entrevistada. A interação ocorreu em ambiente comum a outras prostitutas, o que interferiu na privacidade das informações; contudo, as entrevistadas não demonstraram insatisfação e o plano de intervenção pôde ser satisfatoriamente aplicado. Percebeu-se, nesse momento, a contribuição que a visita trouxe para essas mulheres, uma vez que os apelos de retorno e a satisfação foram explicitados. A cada orientação fornecida, as prostitutas mostravam interesse, indagavam acerca de mais informações e explicitavam o que era ou não possível ser realizado.

No terceiro encontro, verificou-se a implementação das intervenções, bem como avaliou-se o grau de dificuldade para a adesão às ações de autocuidado. Percebeu-se que as mesmas reconheciam a importância das intervenções, mas não cumpriram todas elas. Novas orientações foram feitas, adaptadas aos novos desafios, o que trouxe benefícios para o empoderamento das mesmas.

\section{DISCUSSÃO}

Os pressupostos que perpassaram a análise e interpretação dos dados coletados estavam relacionados à condição intelectual, motivação para mudança de comportamento e falta de apoio na tomada de decisões. Para a realização de uma efetiva prática de autocuidado, deve-se descrever o ambiente e o paciente a fim de identificar fatores significativos para a adoção do novo comportamento ${ }^{(9)}$.

Conforme foi percebido, o principal problema encontrado referiu-se à ausência de rotina diária de exercícios físicos, justificada pela maioria por falta de tempo livre e pela inconstância no horário de trabalho. Compreende-se a importância da prática de atividades físicas para ter uma boa qualidade de $v_{i d a}{ }^{(18)}$. A atividade física é uma orientação humana básica essencial durante toda a vida. Sofre influência de uma série de fatores, com ênfase nos aspectos físicos e psicológicos(19). Dessa forma, emergiu a necessidade de realizar orientações quanto à importância da prática de atividades físicas a todas as prostitutas, com boa aceitação, porém baixa adesão.

Outra alteração encontrada que poderia prejudicar o autocuidado esteve relacionada à alimentação, que, por sua vez, mostrou qualidade deficiente. A alimentação sofre influência de diversos fatores externos ${ }^{(20)}$, como os socioculturais. Estudo realizado mostrou que poucas participantes mencionaram a manutenção de cuidados com a alimentação. Na prostituição, a dificuldade de manter uma boa alimentação pode estar associada não somente às dificuldades financeiras, mas à aceitação da comida disponível nos ambientes de trabalho, como a rua ou as casas fechadas, que não oferecem uma alimentação considerada saudável(21).

No presente estudo, o argumento utilizado foi a falta de tempo para as refeições, em ambas as realidades de prostituição. As diretrizes básicas para uma boa alimentação envolvem todos os grupos alimentares e ressaltam a qualidade dos produtos ingeridos. Embora seja indicado o consumo de todos os grupos de alimentos, alguns, como açúcares, sal e gorduras, devem ter ingestão moderada(22).

O risco para aquisição de infecções, como as DST/Aids, corroborou alguns achados da literatura, principalmente no concernente ao parceiro de risco, o fixo. Estudos mostram que a relação sexual protegida é mais comum com os clientes quando comparada à com o parceiro fixo ${ }^{(23,24)}$. Esse fato é explicado pelas manifestações de insatisfação do companheiro ou pela simbologia da diferenciação entre as práticas profissionais ou afetivas ${ }^{(21)}$.

A baixa autoestima e a desmotivação para a mudança comportamental também foram detectadas durante a visita domiciliar. Esses fatores comumente acometem as prostitutas, principalmente pela falta de perspectiva dentro da profissão. No início da prostituição, as perspectivas e ilusões provocadas parecem uma boa oportunidade de melhorar as condições de vida. Porém, após anos de trabalho, essas ilusões são desfeitas e a realidade de vida mostra sua verdadeira face: as mulheres têm uma rede social de apoio precária, as condições financeiras não melhoraram e o estigma da sociedade mantém-se ${ }^{(21)}$.

Estudo desenvolvido em Porto Alegre mostrou uma alta prevalênciadesintomas depressivoseasanálisesevidenciaram associação entre presença de sintomas depressivos e uso de álcool, história de doenças sexualmente transmissíveis e falta de algum tipo de prática religiosa ${ }^{(25)}$. Autores afirmam que os sintomas depressivos de prostitutas estão associados à utilização de fármacos, álcool e isolamento, além do sentimento de desvalorização. Percebe-se o preconceito vivenciado por vizinhos, familiares e sociedade, acrescido da trajetória de sofrimento e vazio espiritual(26). 
Orem identificou, em sua teoria, que, para atender aos requisitos de autocuidado do indivíduo, são necessários três sistemas de enfermagem: o sistema totalmente compensatório, o sistema parcialmente compensatório e o sistema de apoio-educação. O sistema de apoio-educação caracteriza-se quando um indivíduo consegue executar, ou pode e deve aprender a executar medidas de autocuidado. $O$ mesmo é capaz de regular o exercício e o desenvolvimento de suas atividades de autocuidado. $O$ enfermeiro tem como objetivo promover esse indivíduo a um agente capaz de se cuidar ${ }^{(10)}$. Esse sistema foi o utilizado em nosso estudo, visto que as mulheres tinham toda a capacidade para aprender ou intensificar seu autocuidado.

\section{CONCLUSÃO}

A partir da utilização da Teoria de Orem e da aplicação do processo de enfermagem em prostitutas, constatou-se uma maior facilidade na abordagem do indivíduo, facilitando a interação e a deteç̧ão das alterações nos requisitos de autocuidado. Ademais, a elaboração do plano de cuidados em conformidade com as pacientes permitiu maior adesão às orientações e proporcionou uma valorização das necessidades prioritárias.

A partir das intervenções baseadas no sistema apoioeducação, pôde-se contribuir para o desenvolvimento da capacidade de execução do autocuidado, com conhecimento e maior segurança. Alguns fatores impediram maior contribuição dessa interação, como a falta de privacidade, a rotatividade das mulheres nos serviços, a instabilidade de horários e o tempo de interação, limitado a três visitas. Porém, ressalte-se que o sucesso das intervenções se deve ao interesse e à capacidade do indivíduo em seu cuidado.

Este estudo é de grande valia para o estado da arte nessa temática, contribuindo assim para o maior conhecimento das especificidades dessa população, bem como um futuro auxílio à execução de novas intervenções nessa clientela, utilizando modelos próprios da enfermagem em populações maiores.

\section{Referências}

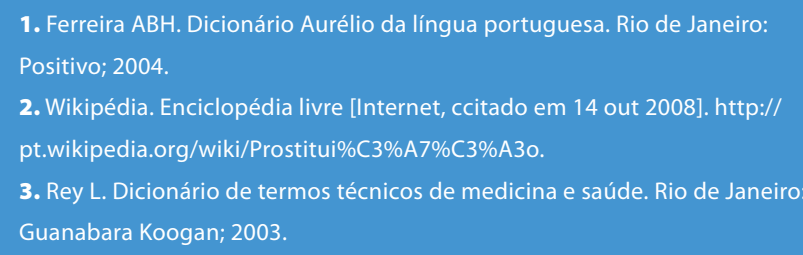

14. Gil AC. Como elaborar projetos de pesquisa. São Paulo: Atlas; 2002.

15. North American Nursing Diagnosis Association. Diagnósticos de enfermagem da Nanda: definições e classificação 2007-2008. Porto Alegre: Artmed; 2008.

16. McCloskey JC, Bulechek GM. Nursing Interventions Classification (NIC). St. Louis: Mosby; 2000.

17. Silva JG, Gurgel AA, Frota MA, Vieira LJES, Valdés MTM. Promoção da saúde: possibilidade de superação das desigualdades sociais. Rev Enferm Uerj. 2008;16(3):421-5.

18. Ferreira MS, Najar AL. Programas e campanhas de promoção da atividade física. Ciênc Saúde Coletiva. 2006;10(Supl.):207-19.

19. Roper N, Logan WW, Tierney AJ. Modelo de enfermagem. Portugal: McGrawHill; 1995.

20. Santos LAS. Educação alimentar e nutricional no contexto da promoção de práticas alimentares saudáveis. Rev Nutr Puccamp. 2005;18(5):681-92. 21. Aquino PS. Desempenho das atividades de vida por prostitutas [dissertação]. Fortaleza: Universidade Federal do Ceará; 2007. 22. Ministério da Saúde (BR). Guia alimentar para a população brasileira: promovendo a alimentação saudável. Brasília: Ministério da Saúde; 2006. 23. Passos ADC, Figueiredo JFC. Fatores de risco para DST entre prostitutas e travestis de Ribeirão Preto (SP), Brasil. Rev Panam Salud Publica. 2004;16(2):95-101. 24. Guimarães K, Merchán-Hamann E. Comercializando fantasias: a representação social da prostituição, dilemas da profissão e a construção da cidadania. Rev Estud Fem. 2005;13(3):525-44.

25. Schreiner L, Paim LL, Ramos F, Cunha Filho EV, Martins DM, Silva Junior CL, et al. Prevalência de sintomas depressivos em uma amostra de prostitutas de Porto Alegre. Rev Psiquiatr Rio Gd Sul. 2004;26(1):13-20.

26. Amaya A, Canaval GE, Viáfara E. Estigmatización de las trabajadoras sexuales: influencias en la salud. Colomb Med. 2005;36(3):65-74. 